\title{
ON THIRD-ORDER THREE-POINT RIGHT FOCAL BOUNDARY VALUE PROBLEMS
}

\author{
XIANGYUN WU AND ZHANBING BAI
}

\begin{abstract}
In this paper, a fixed point theorem in a cone, some inequalities of the associated Green's function and the concavity of solutions are applied to obtain the existence of positive solutions of third-order three-point boundary value problem with dependence on the first-order derivative

$$
\begin{gathered}
x^{\prime \prime \prime}(t)=f\left(t, x(t), x^{\prime}(t)\right), \quad 0<t<1, \\
x(0)=x^{\prime}(\eta)=x^{\prime \prime}(1)=0,
\end{gathered}
$$
\end{abstract}

where $f:[0,1] \times[0, \infty) \times R \rightarrow[0, \infty)$ is a nonnegative continuous function, $\eta \in(1 / 2,1)$.

\section{Introduction}

In recent years, there has been much attention focused on questions of positive solutions of two-point, three-point, and multi-point boundary value problems for nonlinear ordinary differential equations, difference equations, and functional differential equations without dependence on the first order derivative, see [1-7, 9, 11-12] and reference therein. It is well known that the famous Krasnosel'skii's fixed point theorem in a cone [8], as well as LeggettWilliams fixed point theorem [10], five functional fixed point theorem [3], they can be regarded as extensions of Krasnosel'skii's fixed point theorem in a cone, play a extremely important role in above study. Anderson et al. [2] has bringed together a variety of recent results about the existence of positive solutions for the third-order three-point boundary value problem without dependence on any lower-order derivatives in fixed point theory. Also, a good set of references was given.

However, all the above works were done under the assumption that the first order derivative is not involved explicitly in the nonlinear term. In this paper, via a generalization of Krasnosel'skii's fixed point theorem in a cone and some inequalities of the associated Green's function for the associated problem, by placing certain restrictions on the nonlinearity, we prove the existence of positive solutions for the third-order three-point boundary value problem with dependence on the first-order derivative

$$
x^{\prime \prime \prime}(t)=f\left(t, x(t), x^{\prime}(t)\right), \quad 0<t<1
$$

Received September 20, 2007.

2000 Mathematics Subject Classification. 34B15.

Key words and phrases. Fixed point theorem in a cone, positive solution, third-order three-point boundary value problem. 


$$
x(0)=x^{\prime}(\eta)=x^{\prime \prime}(1)=0,
$$

where $f:[0,1] \times[0, \infty) \times R \rightarrow[0, \infty)$ is a nonnegative continuous function, $\eta \in(1 / 2,1)$. A solution of (1.1)-(1.2) is nonnegative and concave on $[0,1]$, nondecreasing on $[0, \eta]$, and nonincreasing on $[\eta, 1]$.

As it is pointed out in [2], positive solutions of Problem (1.1) - (1.2) are concave and satisfy some inequality, it was useful in define a cone on which a positive operator was defined, to which a fixed point theorem due to Krasnosel'skii was then applied to yield positive solutions. Readers may find that the property is crucial in defining an appropriate cone in this paper such that a generalization of Krasnosel'skii's fixed point theorem in a cone can be used to obtain positive solutions.

\section{Preliminaries and Lemmas}

Let $X$ is a Banach space and $P \subset X$ a cone. Suppose $\alpha, \beta: X \rightarrow R^{+}$are two continuous nonnegative functionals satisfying

$$
\alpha(\lambda x) \leq|\lambda| \alpha(x), \quad \beta(\lambda x) \leq|\lambda| \beta(x), \quad \text { for } x \in X, \quad \lambda \in[0,1],
$$

and

$$
M_{1} \max \{\alpha(x), \beta(x)\} \leq\|x\| \leq M_{2} \max \{\alpha(x), \beta(x)\}, \quad \text { for } x \in X,
$$

where $M_{1}, M_{2}$ are two positive constants.

Lemma 2.1.([4]) Let $r_{2}>r_{1}>0, L_{2}>L_{1}>0$ are constants and

$$
\Omega_{i}=\left\{x \in X \mid \alpha(x)<r_{i}, \beta(x)<L_{i}\right\}, \quad i=1,2 .
$$

are two open subsets in $X$ such that $\theta \in \Omega_{1} \subset \bar{\Omega}_{1} \subset \Omega_{2}$. In addition, let

$$
\begin{aligned}
& C_{i}=\left\{x \in X \mid \alpha(x)=r_{i}, \beta(x) \leq L_{i}\right\}, \quad i=1,2 ; \\
& D_{i}=\left\{x \in X \mid \alpha(x) \leq r_{i}, \beta(x)=L_{i}\right\}, \quad i=1.2 .
\end{aligned}
$$

Assume $T: P \rightarrow P$ is a completely continuous operator satisfying

$$
\begin{aligned}
\left(S_{1}\right) \quad \alpha(T x) \leq r_{1}, x \in C_{1} \cap P ; & \beta(T x) \leq L_{1}, x \in D_{1} \cap P ; \\
\alpha(T x) \geq r_{2}, x \in C_{2} \cap P ; & \beta(T x) \geq L_{2}, x \in D_{2} \cap P ;
\end{aligned}
$$

or

$$
\begin{array}{rll}
\left(S_{2}\right) \quad \alpha(T x) \geq r_{1}, x \in C_{1} \cap P ; & \beta(T x) \geq L_{1}, x \in D_{1} \cap P ; \\
\alpha(T x) \leq r_{2}, x \in C_{2} \cap P ; & \beta(T x) \leq L_{2}, x \in D_{2} \cap P ;
\end{array}
$$

then $T$ has at least one fixed point in $\left(\bar{\Omega}_{2} \backslash \Omega_{1}\right) \cap P$. 
The corresponding Green's function for the homogeneous problem

$$
\begin{gathered}
x^{\prime \prime \prime}(t)=0, \quad 0 \leq t \leq 1, \\
x(0)=x^{\prime}(\eta)=x^{\prime \prime}(1)=0,
\end{gathered}
$$

is given in [2]

$$
G(t, s)= \begin{cases}\frac{1}{2} s^{2}, & s \leq t, s \leq \eta, \\ \frac{1}{2}\left[t(2 \eta-t)+(t-s)^{2}\right], & \eta \leq s \leq t \\ \frac{1}{2} t(2 s-t), & t \leq s \leq \eta ; \\ \frac{1}{2} t(2 \eta-t), & t \leq s, \eta \leq s .\end{cases}
$$

By [2], if $\eta \in(1 / 2,1)$, there is

$$
G(\eta, s) \geq G(t, s)>0,
$$

for $t \in(0,1], s \in[0,1]$. Thus throughout this paper we assume that $\eta \in(1 / 2,1)$.

Lemma 2.2.([2]) For all $t, s \in[0,1]$,

$$
g(t) G(\eta, s) \leq G(t, s) \leq G(\eta, s)
$$

where

$$
g(t):=\min \left\{\frac{t}{\eta}, \frac{1-t}{1-\eta}\right\}
$$

For conveniens, we give the following Lemma.

Lemma 2.3. For $h \in(0,1-\eta)$, let $\gamma=1-\frac{h}{1-\eta}$, then

$$
\begin{aligned}
& G(t, s) \leq G(\eta, s), \quad \text { for } t, s \in[0,1], \\
& \gamma G(\eta, s) \leq G(t, s), \quad \text { for } s \in[0,1], t \in[\eta-h, \eta+h] .
\end{aligned}
$$

Proof. It follows Lemma 2.2 immediately.

\section{Existence Results of Positive Solutions}

In this section, we obtain positive solutions of (1.1)-(1.2) with use of Lemma 2.1, a generalization of Krasnosel'skii's fixed point theorem of cone expansion and compression and Lemma 2.3.

Problem (1.1)-(1.2) has a solution $x=x(t)$ if and only if $x$ solves the operator equation

$$
x(t)=T x(t):=\int_{0}^{1} G(t, s) f\left(s, x(s), x^{\prime}(s)\right) d s, \quad 0 \leq t \leq 1,
$$

where $G(t, s) \geq 0$ is Green's function for boundary value problem (2.3)-(2.4). 
We let $X=C^{2}[0,1]$ with $\|x\|=\max \left\{\max _{0 \leq t \leq 1}|x(t)|, \max _{0 \leq t \leq 1}\left|x^{\prime}(t)\right|\right\}$ is a Banach space. We seek solutions of (1.1)-(1.2) that lie in a cone, $P$, defined by

$$
P=\left\{x \in X \mid x(t) \geq 0, \text { and } \min _{\eta-h \leq t \leq \eta+h} x(t) \geq \gamma \max _{0 \leq t \leq 1}|x(t)|\right\},
$$

where $\gamma=1-\frac{h}{1-\eta}, h \in(0,1-\eta)$ is a constant.

Define functionals

$$
\alpha(x)=\max _{0 \leq t \leq 1}|x(t)|, \quad \beta(x)=\max _{0 \leq t \leq 1}\left|x^{\prime}(t)\right|, \quad \text { for } x \in X,
$$

then $\alpha, \beta: X \rightarrow R^{+}$are continuous nonnegative functionals, $\|x\|=\max \{\alpha(x), \beta(x)\}$, (2.1), (2.2) hold and

$$
P=\left\{x \in X \mid x(t) \geq 0, \text { and } \min _{\eta-h \leq t \leq \eta+h} x(t) \geq \gamma \alpha(x)\right\} .
$$

Denote

$$
\begin{aligned}
& M=\max _{0 \leq t \leq 1} \int_{0}^{1} G(t, s) d s, \\
& N=\max _{0 \leq t \leq 1} \int_{\eta-h}^{\eta+h} G(t, s) d s, \\
& A=\int_{\eta}^{1}(s-\eta) d s \\
& \bar{A}=\int_{\eta}^{1}(s-\eta) d s, \\
& B=\max \left\{\eta+\int_{0}^{\eta}(\eta-s) d s, \int_{\eta}^{1}(s-\eta) d s\right\} .
\end{aligned}
$$

It is well know that $T: P \rightarrow P$ is completely continuous. In fact, if $x \in P$, by (2.8), there is

$$
\begin{aligned}
\operatorname{Tx}(t) & =\int_{0}^{1} G(t, s) f\left(s, x(s), x^{\prime}(s)\right) d s \\
& \leq \int_{0}^{1} \max _{0 \leq t \leq 1} G(t, s) \cdot f\left(s, x(s), x^{\prime}(s)\right) d s \\
& =\int_{0}^{1} G(\eta, s) \cdot f\left(s, x(s), x^{\prime}(s)\right) d s,
\end{aligned}
$$

so that

$$
\alpha(T x)=\max _{0 \leq t \leq 1}|T x(t)| \leq \int_{0}^{1} G(\eta, s) \cdot f\left(s, x(s), x^{\prime}(s)\right) d s .
$$

Combine with (2.9), we get

$$
\min _{\eta-h \leq t \leq \eta+h} T x(t)=\min _{\eta-h \leq t \leq \eta+h} \int_{0}^{1} G(t, s) f\left(s, x(s), x^{\prime}(s)\right) d s
$$




$$
\begin{aligned}
& \geq \gamma \int_{0}^{1} \max _{0 \leq t \leq 1} G(t, s) \cdot f\left(s, x(s), x^{\prime}(s)\right) d s \\
& \geq \gamma \cdot \alpha(T x) .
\end{aligned}
$$

Also, from the positivity of $G(t, s)$, we have, for $x \in P$, that $T x(t) \geq 0$, for $0 \leq t \leq 1$, and so $T: P \rightarrow P$. Further, standard arguments yields that $T$ is completely continuous.

Theorem 3.1. Suppose there are four constants $r_{2}>r_{1}>0, L_{2}>L_{1}>0$ such that $\max \left\{\frac{r_{1}}{M}, \frac{L_{1}}{A}\right\} \leq$ $\min \left\{\frac{r_{2}}{M}, \frac{L_{2}}{B}\right\}$ and the following assumptions hold

$\left(A_{1}\right) f(t, u, v) \geq \max \left\{\frac{r_{1}}{M}, \frac{L_{1}}{A}\right\}$, for $(t, u, v) \in[0,1] \times\left[0, r_{1}\right] \times\left[-L_{1}, L_{1}\right]$;

$\left(A_{2}\right) f(t, u, v) \leq \min \left\{\frac{r_{2}}{M}, \frac{L_{2}}{B}\right\}$ for $(t, u, v) \in[0,1] \times\left[0, r_{2}\right] \times\left[-L_{2}, L_{2}\right]$.

Then Problem (1.1) - (1.2) has at least one positive solution $x(t)$ such that

$$
r_{1} \leq \max _{0 \leq t \leq 1} x(t) \leq r_{2} \quad \text { or } \quad L_{1} \leq \max _{0 \leq t \leq 1}\left|x^{\prime}(t)\right| \leq L_{2}
$$

Proof. Take

$$
\Omega_{i}=\left\{x \in X \mid \alpha(x)<r_{i}, \beta(x)<L_{i}\right\}, \quad i=1,2 .
$$

are two bounded open subsets in $X$. In addition, let

$$
\begin{array}{ll}
C_{i}=\left\{x \in X \mid \alpha(x)=r_{i}, \beta(x) \leq L_{i}\right\}, & i=1,2 ; \\
D_{i}=\left\{x \in X \mid \alpha(x) \leq r_{i}, \beta(x)=L_{i}\right\}, & i=1.2 .
\end{array}
$$

For $x \in C_{1} \cap P$, by $\left(A_{1}\right)$, there is

$$
\begin{aligned}
\alpha(T x) & =\max _{t \in[0,1]}\left|\int_{0}^{1} G(t, s) f\left(s, x(s), x^{\prime}(s)\right) d s\right| \\
& \geq \frac{r_{1}}{M} \cdot \max _{t \in[0,1]}\left|\int_{0}^{1} G(t, s) d s\right|=r_{1} .
\end{aligned}
$$

According to definition of $T$, we have for $0 \leq t \leq 1$,

$$
\begin{aligned}
& (T x)^{\prime}(t)=(\eta-t) \int_{t}^{1} f\left(s, x(s), x^{\prime}(s)\right) d s+\int_{\eta}^{t}(\eta-s) f\left(s, x(s), x^{\prime}(s)\right) d s, \\
& (T x)^{\prime \prime}(t)=-\int_{t}^{1} f\left(s, x(s), x^{\prime}(s)\right) d s \leq 0 .
\end{aligned}
$$

Thus, $T x$ concave on $[0,1]$, it follows that

$$
\max _{t \in[0,1]}\left|(T x)^{\prime}(t)\right|=\max \left\{\left|(T x)^{\prime}(0)\right|,\left|(T x)^{\prime}(1)\right|\right\} .
$$

Combine with $\left(A_{1}\right)$ and $f \geq 0$, for $x \in D_{1} \cap P$, there is

$$
\beta(T x)=\max _{t \in[0,1]}\left|(T x)^{\prime}(t)\right|
$$




$$
\begin{aligned}
& =\max \left\{\left|(T x)^{\prime}(0)\right|,\left|(T x)^{\prime}(1)\right|\right\} \\
& \geq\left|(T x)^{\prime}(1)\right| \\
& =\int_{\eta}^{1}(s-\eta) f\left(s, x(s), x^{\prime}(s)\right) d s \\
& \geq \frac{L_{1}}{A} \cdot \int_{\eta}^{1}(s-\eta) d s \\
& =\frac{L_{1}}{A} \cdot A=L_{1} .
\end{aligned}
$$

For $x \in C_{2} \cap P$, by $\left(A_{2}\right)$, there is

$$
\begin{aligned}
\alpha(T x) & =\max _{t \in[0,1]}\left|\int_{0}^{1} G(t, s) f\left(s, x(s), x^{\prime}(s)\right) d s\right| \\
& \leq \max _{t \in[0,1]} \int_{0}^{1} G(t, s) \cdot \frac{r_{2}}{M} d s \\
& =\frac{r_{2}}{M} \cdot \max _{t \in[0,1]} \int_{0}^{1} G(t, s) d s=r_{2} .
\end{aligned}
$$

For $x \in D_{2} \cap P$, by $\left(A_{2}\right)$, there is

$$
\begin{aligned}
\beta(T x)= & \max _{t \in[0,1]}\left|(T x)^{\prime}(t)\right| \\
= & \max \left\{\left|(T x)^{\prime}(0)\right|,\left|(T x)^{\prime}(1)\right|\right\} \\
\leq & \max \left\{\eta \int_{0}^{1} f\left(s, x(s), x^{\prime}(s)\right) d s+\int_{0}^{\eta}(\eta-s) f\left(s, x(s), x^{\prime}(s)\right) d s,\right. \\
& \left.\int_{\eta}^{1}(s-\eta) f\left(s, x(s), x^{\prime}(s)\right) d s\right\} \\
\leq & \frac{L_{2}}{B} \cdot B=L_{2} .
\end{aligned}
$$

Now, Lemma 2.1 implies there is $x \in\left(\bar{\Omega}_{2} \backslash \Omega_{1}\right) \cap P$ such that $x=T x$, namely, Problem $(1.1)-(1.2)$ has at least one positive solution $x(t)$ such that

$$
r_{1} \leq \alpha(x) \leq r_{2} \quad \text { or } \quad L_{1} \leq \beta(x) \leq L_{2}
$$

i.e.,

$$
r_{1} \leq \max _{0 \leq t \leq 1} x(t) \leq r_{2} \quad \text { or } \quad L_{1} \leq \max _{0 \leq t \leq 1}\left|x^{\prime}(t)\right| \leq L_{2} .
$$

The proof is complete.

Theorem 3.2. Suppose there are five constants $0<h<1-\eta, 0<r_{1}<r_{2}, 0<L_{1}<L_{2}$ such that $\max \left\{\frac{r_{1}}{N}, \frac{L_{1}}{\bar{A}}\right\} \leq \min \left\{\frac{r_{2}}{M}, \frac{L_{2}}{A}\right\}$ and the following assumptions hold

$\left(A_{3}\right) f(t, u, v) \geq \frac{r_{1}}{N}, f o r(t, u, v) \in[\eta-h, \eta+h] \times\left[\gamma r_{1}, r_{1}\right] \times\left[-L_{1}, L_{1}\right]$ 
$\left(A_{4}\right) f(t, u, v) \geq \frac{L_{1}}{\bar{A}}$, for $(t, u, v) \in[0,1] \times\left[0, r_{1}\right] \times\left[-L_{1}, L_{1}\right]$;

( $\left.A_{5}\right) f(t, u, v) \leq \min \left\{\frac{r_{2}}{M}, \frac{L_{2}}{A}\right\}$, for $(t, u, v) \in[0,1] \times\left[0, r_{2}\right] \times\left[-L_{2}, L_{2}\right]$.

Then Problem (1.1) - (1.2) has at least one positive solution $x(t)$ such that

$$
r_{2} \leq \max _{0 \leq t \leq 1} x(t) \leq r_{1} \quad \text { or } \quad L_{2} \leq \max _{0 \leq t \leq 1}\left|x^{\prime}(t)\right| \leq L_{1}
$$

Proof. We just need notice the following difference to the proof of Theorem 3.1. For $x \in C_{1} \cap P$, the definition of $P$ implies that

$$
x(t) \geq \gamma \alpha(x)=\gamma r_{1}, \quad \text { for } t \in[\eta-h, \eta+h] .
$$

By $\left(A_{3}\right)$, there is

$$
\begin{aligned}
\alpha(T x) & =\max _{t \in[0,1]}\left|\int_{0}^{1} G(t, s) f\left(s, x(s), x^{\prime}(s)\right) d s\right| \\
& \geq \max _{t \in[0,1]}\left|\int_{\eta-h}^{\eta+h} G(t, s) f\left(s, x(s), x^{\prime}(s)\right) d s\right| \\
& \geq \max _{t \in[0,1]}\left|\int_{\eta-h}^{\eta+h} G(t, s) \cdot \frac{r_{1}}{N} d s\right| \\
& =r_{1} .
\end{aligned}
$$

For $x \in D_{1} \cap P$, by $\left(A_{4}\right)$, there is

$$
\begin{aligned}
\beta(T x) & =\max _{t \in[0,1]}\left|(T x)^{\prime}(t)\right| \\
& =\max \left\{\left|(T x)^{\prime}(0)\right|,\left|(T x)^{\prime}(1)\right|\right\} \\
& \geq\left|(T x)^{\prime}(1)\right| \\
& =\int_{\eta}^{1}(s-\eta) f\left(s, x(s), x^{\prime}(s)\right) d s \\
& \geq \frac{L_{1}}{\bar{A}} \cdot \int_{\eta}^{1}(s-\eta) d s \\
& =\frac{L_{1}}{\bar{A}} \cdot \bar{A}=L_{1} .
\end{aligned}
$$

The rest of proof is similar to Theorem 3.1 and the proof is complete.

\section{References}

[1] R. P. Agarwal, D. O’Regan and P. J. Y. Wong, Positive Solutions of Differential, Difference, and Integral Equations, Kluwer Academic Publishers, Boston, 1999.

[2] D. R. Anderson and J. M. Davis, Multiple solutions and eigenvalues for third order right focal boundary value problems, J. Math. Anal. Appl. 267(2002), 135-157. 
[3] R. I. Avery, A generalization of the Leggett-Williams fixed point theorem, Math. Sci. Res. Hot-line 2(1998), 9-14.

[4] Z. B. Bai and W. G. Ge, Existence of positive solutions to fourth-order quasilinear boundary value problems, Acta. Math. Sinica. 22(2006), 1825-1830.

[5] Z. J. Du, W. G Ge and X. J. Lin, Existence of solutions for a class of third-order nonlinear boundary value problems, J. Math. Anal. Appl. 294(2004), 104-112.

[6] Z. J. Du, W. G Ge and M. R. Zhou, Singular perturbations for third-order nonlinear multi-point boundary value problem, J. Differential Equations 218(2005), 69-90.

[7] L. H. Erbe and H. Y. Wang, On the existence of positive solutions of ordinary differential equations, Proc. Amer. Math. Soc. 120(1994), 743-748.

[8] D. Guo and V. Lakshmikantham, Nonlinear Problems in Abstract Cones, Academic Press, New York, 1988.

[9] J. Henderson and H. Y. Wang, Positive solutions for nonlinear eigenvalue problems, J. Math. Anal. Appl. 208(1997), 252-259.

[10] R. W. Leggett and L. R. Williams, Multiple positive fixed points of nonlinear operators on ordered Banach spaces, Indiana Univ. Math. J. 28(1979), 673-688.

[11] R. Y. Ma, Positive solutions of nonlinear three-point boundary value problem, Electronic J. Diff. Equa. 34(1999), 1-8.

[12] Q. L. Yao and Y. Q. Feng, The existence of a third-order two-point boundary value problem, Appl. Math. Lett. 15(2002), 227-232.

Department of Mathematics and physics, Lijiang Teacher's College, Lijiang, 674100, P.R. China.

E-mail: ljwxy@126.com

Institute of Mathematics, Shandong University of Science and Technology, Qingdao, 266510, P.R. China.

E-mail: zhanbingbai@163.com 